\title{
MRI IN PSEUDOTUMOR CEREBRI
}

The MRI has been used to clarify the pathophysiology of pseudotumor cerebri in seven children, aged between six months and 13 years, reported from British Columbia's Children's Hospital, Vancouver. All had papilledema, three abducens palsies, and six had headache and/or visual impairments. Topical steroid therapy for eczema and mastoiditis were predisposing factors in two. CT head scans were normal. MRIs showed normal signal intensity in the white matter, suggesting that periventricular brain water content was not markedly increased. The authors hypothesize an equilibrium between raised CSF outflow resistance and increased cerebral blood volume and/or mild interstitial cerebral edema. (Connolly MB et al. Magnetic resonance imaging in pseudotumor cerebri. Dev Med Child Neurol Dec 1992; 34: 1091-1094). (Correspondence: Dr Kevin Farrell, Rm 1D14, British Columbia's Children's Hospital, 4480 Oak St, Vancouver, B.C. V6H 3V4).

COMMENT. Increased cerebral blood volume, confirmed by PET, an abnormal CSF dynamics, demonstrated by decreased arachnoid absorption, and cerebral edema, defined by brain biopsy, are some of the hypotheses proposed in pseudotumor cases. Periventricular edema, the expected consequence of a resistance to CSF outflow, was not demonstrated in this MRI study. Predisposing factors in children have included otitis media, venous sinus thrombosis, mastoiditis, mild head trauma, viral infections, vitamin A intoxication, teracyclines, obesity, menstrual irregularities, nutritional disturbances, and steroid therapy or withdrawal.

\section{KNEE-CHEST POSITION: A SIGN OF INCRFASED IC PRESSURE}

A preference to lie in the knee-chest position was associated with increased intracranial pressure (ICP) in three children, ages 12 and 20 months and 4 years, reported from the Sackler School of Medicine, Tel Aviv University, Petach Tikvah, Israel. The forehead touched the floor on a level with the chest and knees, and the rest of the trunk was elevated so that the buttocks were uppermost. The 4-year old child had a mass in the pineal area, with obstructive hydrocephalus; the 20 month-old had pseudotumor cerebri that responded to acetazolamide and a return to a normal posture; the 12-month old had a cerebellar astrocytoma and obstructive hydrocephalus. The authors postulate that the knee-chest position favors a reduced pressure in the right heart atrium, thus augmenting the blood flow from the superior vena cava and dural sinuses. (Straussberg R et al. Knee-chest position as a sign of increased intracranial pressure in children. LPediatr Jan 1993; 122: 99-100). (Reprints: Rachel Straussberg MD, Dept Pediatrics, Hasharon Hospital, Petach Tikvah, Israel). 
COMMENT. In infants and young children with brain tumors and few signs or subjective complaints, the knee-chest posture might be helpful in the clinical suspicion and diagnosis of increased intracranial pressure. This posture is also assumed by children with cyanotic heart disease and is a characteristic sign of acrodynia.

\section{DEGENERATIVE DISEASES}

\section{FOCAL TUMOR-LIKE DEMYELINATING LESIONS}

Thirty-one patients with large, focal cerebral demyelinating lesions are reported from the University of Kansas School of Medicine, Kansas City. The lesions presented clinically and radiologically as brain tumors or as multiple cysts. The demyelinating pathology was established through biopsy and a significant improvement with corticosteroid therapy. Four patients were children, ages 8 - 12 years, and one was an adolescent, aged 18 . One young adult had received an influenza vaccine 10 days before the onset of symptoms. The clinical course favored postinfectious/postvaccination encephalitis, but the tumor-sized masses of demyelination were atypical. (Kepes JJ. Large focal tumor-like demyelinating lesions of the brain: intermediate entity between multiple sclerosis and acute disseminated encephalomyelitis? A study of 31 patients. Ann Neurol Jan 1993; $\underline{33}$ : 18-27). (Correspondence: Dr Kepes, Department of Pathology and Oncology, University of Kansas College of Health Sciences and Ilospital, 39th and Rainbow Blvd, Kansas City, KS 66103).

COMMENT. The author rules out Schilder's disease, a progressive childhood diffuse sclerosis, which often presents with asymmetrical, large focal hemispheric lesions and smaller lesions, sometimes difficult to differentiate from multiple sclerosis. ( See Greenfield's Neuropathology, Baltimore, Williams and Wilkins, 1963). Many reported cases of Schilder's disease are now thought to represent either adrenoleukodystrophy or acute forms of multiple sclerosis (Menkes JH. Textbook of Child Neurology. 3rd ed. Philadelphia, Lea \& Febiger, 1985).

\section{MRI IN INFANTILE NEUROAXONAL DYSTROPHY}

The MRI findings in four children aged 3 to 10 years with infantile neuroaxonal dystrophy are reported from the Chiba Children's Hospital, Japan. $\mathrm{T}_{2}$ - weighted images showed bilateral diffuse hyperintensity of the cerebellar cortex and cerebellar atrophy. Autopsy changes in one patient included cerebellar atrophy, ventricular dilatation, spheroids in the gray matter, loss of neurons, axonal swellings, and extensive astrogliosis. (Tanabe $Y$ et al. The use of magnetic resonance imaging in diagnosing infantile neuroaxonal dystrophy. Neurology Jan 1993; 43: 110-113). (Reprints: Dr Y Tanabe, Division of Neurology, Chiba Children's Hospital, 579-1 Heta-cho, Midori-ku, Chiba 266, Japan). 